\title{
A theoretical prediction of the effects of electrostatic forces on saltating snow particles
}

\author{
Scott Schmidt and J. D. Dent \\ Department of Civil and Agricultural Engineering, Montana State University, Bozeman, MT 59715, U.S.A.
}

\begin{abstract}
Electrification of snow particles during blizzards creates forces that may strongly effect their trajectories in saltation along a charged surface. This paper shows the results of adding such forces to the equations of motion for saltation. Evaluating these equations requires knowledge of the electric-field strength very near the surface, and a review shows that such measurements are lacking. However, extrapolation of reported measurements from greater heights agrees with the electric field computed for a bed of spherical particles. In this hypothetical field, the equations of motion predict large changes in saltation length that might occur as particles pass over surfaces with positive and negative sign. Such changes apparently coincide with surface erosion and deposition.
\end{abstract}

\section{INTRODUCTION}

Saltation is a transport mechanism by which solid particles hop along a surface in a fluid shear flow. Whether it be snow or sand particles in air, sediment in a river bed, or sand in a density current on the ocean floor, the equations that describe this motion are of similar form. The bounce of a particle, rebounding from elastic impact with the surface, is stretched into a long, low trajectory responding to forces of fluid drag and gravitation. Lift forces also develop if the particle spins (White and Schultz, 1977).

Boundary surfaces in drifting snow acquire electrostatic charge, and electric fields in blizzards are often several orders of magnitude stronger than without drifting (e.g. Pearce and Currie, 1949), as will be explained. The drift particles also develop a charge, usually of negative sign. Improving the mathematical description of saltating snow by including electrostatic forces may help predict and control problems such as ice build-up on roads and runways during ground blizzards. Including these forces in the saltation equations should also aid interpretation of wind-tunnel simulations of both drifting sand and snow, which are often confounded by electrostatic charge.

In this paper, a review of electrostatic measurements made in drifting snow, and of the physical basis for this charge generation and separation, leads to a proposed set of saltation equations that include electrostatic forces. A hypothesis regarding the electric field in the saltation region results from comparing the theoretical electric field above a bed of ice spheres with measured fields at greater heights in drifting snow, and a numerical solution of the equations provides example trajectories.

\section{THE SALTATION PROCESS IN SNOW}

In describing the motion of sand particles in water, Gilbert (1914) used the word saltation to describe the hopping motion of sand particles near the flow bed. Later, Bagnold (1941) showed that this same mechanism occurred in wind-blown sand, and Kobayashi (1972) first photographed saltation occurring in drifting snow. Saltation accounts for almost all mass transport in ground blizzards, and provides the source of particles for suspended transport in more intense storms.

Saltation of snow in air occurs when wind across a snow surface exceeds some threshold speed to dislodge particles and eject them into the flow. Once dislodged, the snow particles bounce along the boundary, within a few centimeters of the surface. Photographs show that the particles are often rounded by abrasion, with equivalent spherical diameters ranging from 0.1 to $0.5 \mathrm{~mm}$ (Schmidt, 1981). Kobayashi (1972) estimated trajectory lengths as large as $1 \mathrm{~m}$ in moderate drifting, with mean lengths between 0.1 and $0.3 \mathrm{~m}$ for winds speeds of $10 \mathrm{~m} \mathrm{~s}^{-1}$ at $1 \mathrm{~m}$ height.

The restitution coefficient of an ice sphere bouncing on an ice block ranges from 0.6 to 0.9 as temperature decreases from $-5^{\circ}$ to $-20^{\circ} \mathrm{C}$ (Kobayashi, 1972). Impacts of saltating snow are sufficient to fracture ice bonds holding surface grains (Schmidt, 1980), and impact energy may be transferred to several new particle trajectories. This rebounding of particles on the surface transfers significant momentum from the atmosphere to the planetary boundary (Kikuchi, 1981), and presents a drag on the wind that is reflected in the vertical velocity profile (Maeno and others, 1979). 


\section{ELEGTRIFICATION OF BLOWING SNOW}

Many observers have reported electrification of natural blowing snow. Simpson (1919), British National Committee for the Polar Year (1937), Schaefer (1947), Pearce and Currie (1949), Barré (1953), and Magono and Sakurai (1963) all observed a considerable increase in the positive electric-field gradient normal to the Earth's surface, during blowing and drifting snow events. Measurements in Antarctica by Wishart and Radok (1967) and Wishart (1968) showed that blowing snow particles develop negative charges. Latham and Montagne (1970) also showed negatively charged snow particles moving in a positive electric field that increased as the roof of a snow cornice was approached.

\section{Charge separation in ice}

Efforts to explain electrification in thunderstorms have produced most of the knowledge on charge separation mechanisms in ice. Reynolds and others (1957) hypothesized that impacts of ice crystals with a graupel pellet would create a liquid film by pressure contact, and that freezing potentials produced charge separation during regelation.

Latham and Mason (1961) demonstrated that charge separation in ice resulted from temperature gradients. Hobbs (1974, p. 179) reviews the theory of this process, termed the thermoelectric effect. Concentrations of $\mathrm{H}^{+}$ and $\mathrm{OH}^{-}$ions increase as the temperature on an ice specimen increases. $\mathrm{H}^{+}$ions have greater mobility in the ice lattice than $\mathrm{OH}^{-}$ions, therefore they migrate more rapidly along a concentration gradient. When a temperature gradient in the ice produces an ionic concentration gradient, $\mathrm{H}^{+}$ions move more rapidly to colder regions. The process develops a potential difference across uniform pure ice of $2.3 \pm 0.3 \mathrm{mV}$ for each ${ }^{\circ} \mathrm{C}$ of temperature difference (Hobbs, 1974).

Latham and Mason (1961) extended this theory to two separate ice specimens. When two pieces of ice at different temperatures were brought into contact for a short period of time and then separated, the warmer piece of ice acquired a negative charge while the cooler piece became positively charged.

\section{Electrification in blizzards}

From laboratory experiments on the electrification of wind-blown snow, Latham and Stow (1967) suggested three primary processes for charge separation, all involving the thermoelectric effect: crystal fragmentation, asymmetric rubbing, and transient contact of blown particles with the surface. Each is described below. They found that contributions to electrification by the Workman-Reynolds effect and by evaporation of ice were minimal.

Crystal fragmentation separates charge when a temperature gradient exists across the crystal. Such a gradient might exist when the temperature of the wind differs from the snow temperature below the surface. If the wind is warmer, the most exposed projections on the surface are warmed and become negatively charged. If such a fragment breaks from the surface, it carries a net negative charge.
Asymmetric rubbing refers to a difference in contact areas when two ice pieces rub together. If a small ice crystal slides along a larger ice surface, the contact area of the crystal remains small, while the area contacted on the surface increases with time. Greater frictional heating of the crystal results, and the warmer crystal gains a negative charge. Henry (1953) described charging of insulators by asymmetric rubbing, which was demonstrated with ice by Reynolds and others (1957), and by Latham (1963).

In the third process, if a temperature gradient exists over the areas of contact, charge will be transferred during the transient contact of a blown particle with the surface. If air flow is warmer than the surface, particles receive negative charge during contact since surface particles have negatively charged extremities, as in the crystal fragmentation process. Net charge on the surface becomes opposite in sign. The signs are reversed if colder air creates saltation across a warmer surface.

A fourth process suggested by Latham and Stow (1967), ionization of air by point discharge as highly charged particles rebound from the surface, depends on the amount of charge separation developed by the first three. Stronger wind velocity increases charge separation (Latham and Miller, 1965), increasing production of ions by point discharge. As Hobbs (1974) pointed out, the relative importance of these processes under natural conditions remained to be determined. Apparently this is still the case.

Other theories explain charge separation in thunderstorms without the thermoelectric effect. Scott and Levin (1970) described polarization of ice particles by a potential gradient, which enhances charge separation during collisions with smaller ice crystals. The force of the collision between the particles and roughness of the ice surfaces are also factors. Scott and Hobbs (1968) showed that the sign of the charge acquired by an ice specimen from a collision depends on the shapes of the particles involved and the magnitude of the charge acquired increases with increased impact velocities. Latham and Stow (1965) found that charge transfer associated with the thermoelectric effect could be greatly enhanced if the ice specimens are rough and if impact velocities are high.

\section{Electrostatic force on saltating particles}

From work done in a laboratory setting, Latham and Stow (1967) deduced that the maximum possible charge, $Q$, that a particle of size $L$ could support was approximately

$$
Q \approx 10^{-5} L^{2}
$$

where units of $Q$ are picocoulombs and $L$ is in meters (see also Latham and Montagne, 1970). In field experiments, Latham and Montagne (1970) found the charge on natural particles saltating over a cornice roof was very near this maximum theoretical value.

Maeno and others (1985) showed that negative charge also develops on snow particles saltating in a wind tunnel. The charge on surface particles varied from $-0.01 \mu \mathrm{C} \mathrm{kg}^{-1}$ to $+0.03 \mu \mathrm{C} \mathrm{kg}^{-1}$. These magnitudes were near those of the saltating particles, and sign changes appeared to coincide with local deposition and erosion areas on the surface. These researchers attributed charge-to-mass 

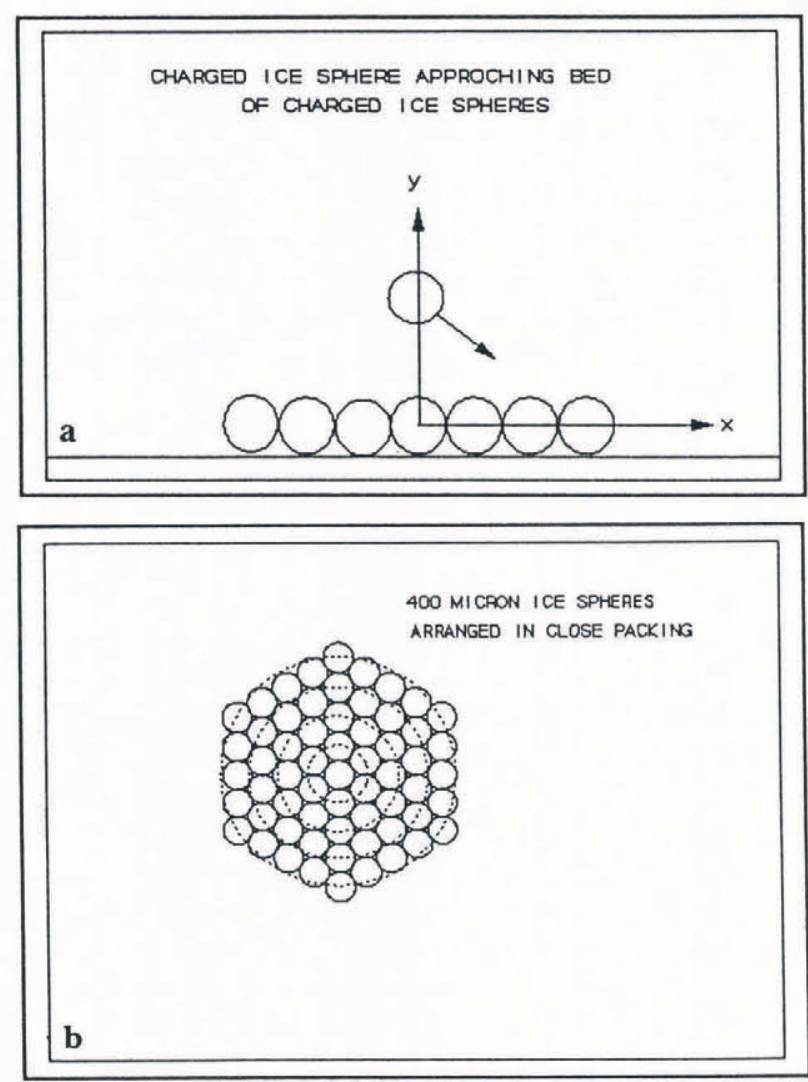

Fig. 1. Defining sketch for equations describing twodimensional motion of a sphere saltating on a bed of charged spheres: $a$, the coordinate system, with $x$ extending downwind, $y$ normal to the surface; $b$, plan view of 10 rings of six spheres, hexagonally packed, from which electric field normal to the surface was computed.

ratios lower than those seen in natural blowing snow by Latham and Montagne (1970) and Wishart (1968) to the short fetch distance of their wind tunnel.

\section{Estimating electric field strength}

The problem in estimating the electrostatic force on a saltating snow particle is a lack of electric-field measurements in the saltation region, within the few centimeters nearest the surface. The following model was used to estimate this field. Based on wind-tunnel experiments by Maeno and others (1985), the charge-to-mass ratio of surface particles was assumed equal in magnitude, but opposite in sign, to that measured on saltating particles. Consider a charged ice sphere saltating over a uniform bed of similar spheres (Fig. la). The electric field above the bed can be calculated by treating each particle as a point charge and summing contributions to the field surrounding the moving particle. Such computations are simplified by adding rings of six spheres (hexagonal packing) equidistant from the bed particle closest to the moving particle (Fig. 1b). Contributions to the field decrease rapidly as the radius of these rings increases, so that little is contributed beyond 10 rings, or the area covered by 61 bed spheres.

As an example, such calculations were made for spheres with $400 \mu \mathrm{m}$ diameter, the average equivalent diameter in experiments by Latham and Montagne (1970). Two values of particle charge-to-mass ratio were used: $-10 \mu \mathrm{C} \mathrm{kg}^{-1}$ (Latham and Montagne, 1970) and
$-50 \mu \mathrm{C} \mathrm{kg}^{-1}$ (Wishart, 1968, data for 9 January 1968).

Latham and Montagne (1970) also measured the electric field above three cornices where they found the Efield varied significantly with distance from the surface of the snow. A comparison of the electric field computed for an incoming saltation particle above an ice-sphere bed to the field extrapolated from Latham and Montagne's (1970) field measurements is shown in Figure 2. The points on the line labeled "combined" were chosen to fit the cornice data at distances far from the saltation surface and the computed electric field at distances close to the surface. The regression line through these points represents our best estimate of electric field as a function of height above the saltation surface.

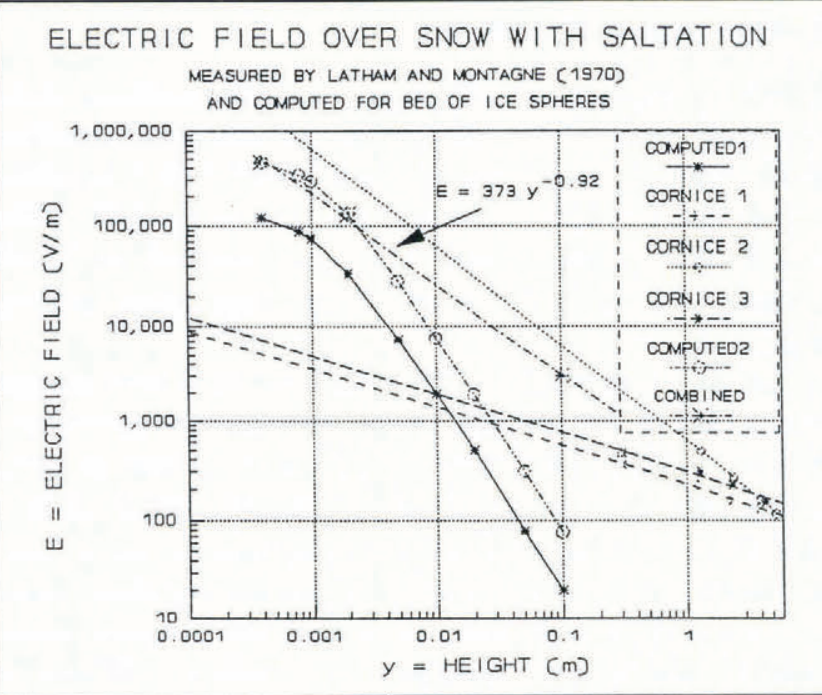

Fig. 2. A plot of electric-field strength as a function of distance normal to the charged boundary. The power-law profile marked "combined" was chosen for computations by extrapolating measured values (Latham and Montagne, 1970) to meet values computed for a bed of charged spheres (Fig. 1).

\section{SALTATION EQUATIONS}

The saltation equations developed by White and Schultz (1977) consider four forces acting on a spherical particle. The first is the force on the particle due to the motion of the fluid, the second is the resistance to the fluid motion due to drag, the third is the gravitational force, and the fourth is a lift force due to Magnus effects. The horizontal, vertical and rotational components of the equation of motion are written as

$$
\begin{aligned}
m_{\mathrm{p}} \ddot{x} & =L \frac{\dot{y}}{V_{\mathrm{r}}}-D \frac{\dot{x}-u}{V_{\mathrm{r}}}, \\
m_{\mathrm{p}} \ddot{y} & =-L \frac{\dot{x}-u}{V_{\mathrm{r}}}-D \frac{\dot{y}}{V_{\mathrm{r}}}-m_{\mathrm{p}} g, \\
I_{\mathrm{p}} \ddot{\Theta} & =M,
\end{aligned}
$$

where $m_{\mathrm{p}}$ is the mass of the particle, $u$ is the velocity of the fluid as a function of height above the surface, $I_{\mathrm{p}}$ is the particle's moment of inertia, and $\ddot{\Theta}$ is the particle's angular acceleration. $V_{\mathrm{r}}$ is the velocity of the particle relative to the fluid flow, and its magnitude can be expressed as 


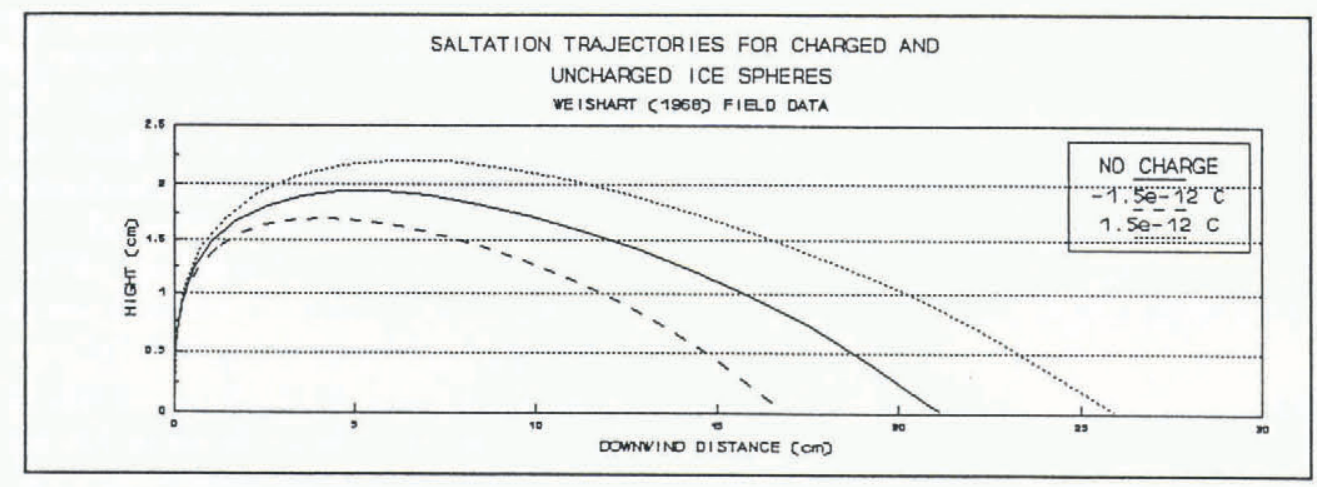

Fig. 3. Saltation trajectories computed with and without electrostatic forces, for a $400 \mu \mathrm{m}$ diameter ice sphere. Assumed particle charge-to-mass ratio was taken from Wishart's (1968) field data as $50 \mu \mathrm{Ckg}^{-1}$.

$$
V_{\mathrm{r}}=\left[(\dot{x}-u)^{2}+\dot{y}^{2}\right]^{\frac{1}{2}} .
$$

The drag force, $D$, is expressed in terms of the drag coefficient, $C_{\mathrm{d}}$, as

$$
D=\frac{1}{2} C_{\mathrm{d}} A_{\mathrm{p}} \rho V_{\mathrm{r}}^{2},
$$

where $A_{\mathrm{p}}$ is the cross-section area of the particle.

The lift force, $L$, is dependent on the spinning motion of the particle. If the particle is assumed to be a rotating sphere, then the equations describing its rotational motion are a set of coupled first-order equations given by

$$
\begin{aligned}
L & =\frac{1}{8} \pi D^{3} \rho V_{\mathrm{r}}\left(\dot{\Theta}-\frac{1}{2} \partial u / \partial y\right), \\
M & =\pi \mu D^{3}\left(\dot{\Theta}-\frac{1}{2} \partial u / \partial y\right),
\end{aligned}
$$

where $\dot{\Theta}$ is the particle's angular velocity and $\mu$ is the fluid viscosity.

To investigate the effects that an electrostatic force would have on saltation trajectories, we add the force on a charged particle in an electric field,

$$
F=q E,
$$

where $E$ is the electric field vector and $q$ is the charge on the particle. This electrostatic force would act normal to the saltation surface. For initial calculation, the $x$ axis is taken on the saltation surface, along the wind direction. The net electrostatic force acts only in the vertical, along the $y$ axis (Fig. 1a). The equation that describes the particle's vertical component of motion would take the form

$$
m_{\mathrm{p}} \ddot{y}=-L \frac{\dot{x}-u}{V_{\mathrm{r}}}-D \frac{\dot{y}}{V_{\mathrm{r}}}+q E-m_{\mathrm{p}} g .
$$

With the assumption that the charge on the saltating particle remains constant through one saltation trajectory, using the combined relationship (Fig. 2) for the electric field above a uniform bed of ice spheres produces equations of motion that can be solved using numerical methods. An Adams fourth-order predictor-corrector procedure was used to solve the set of equations. Figure 3 shows example trajectories for both positive and negative charge-to-mass ratios from Wishart's (1968) field data, and compares these to the trajectory of a particle with no charge.

\section{DISCUSSION}

Evidence in the literature indicates that potential gradients much greater than the fair weather gradient develop above a surface in blowing snow. Further, saltating snow particles develop large electrostatic charges, usually of negative sign. The inclusion of electrostatic forces in the equations describing saltation of charged particles in a strong electric field predict a large influence on saltating particle trajectories. Using charge-to-mass ratios found by Wishart (1968), computed trajectory lengths were altered by as much as $24 \%$ from those predicted for uncharged particles.

Electrostatic forces might play an important role in many areas. Latham and Montagne (1970) directed their experiment at determining the role electrostatics might play in formation of snow cornices. Based on the electric fields measured at distances no closer than $30 \mathrm{~cm}$ above the cornices, they concluded that such forces might explain certain aspects of cornice build-up, especially at low wind speeds. They recommended "considerable further study", which seems warranted in view of calculations presented here.

The mechanism offers an alternative explanation for snow ripple and dune formation. Consider a charged particle moving over a surface with alternating sign, such as might occur over regions of erosion and deposition that extend transverse to the wind. Eroding surfaces would acquire charge of sign opposite that of the saltating particle, while deposition areas would hold the same charge sign as the incoming particle. Trajectories over the deposition area would be longer, with lower impact angle, than trajectories on the eroding surface. The particle would experience a decrease in the vertical component of the rebound, leading to decay of the saltation motion in the deposition region.

Electrostatic forces might offer a first explanation for the winding path of particle flow, called "snow-snakes", that often occur in ground blizzards at low wind speeds. If the hypothesis presented in this paper was extended to three dimensions, a negatively charged particle approaching a surface area of positive charge would experience an attractive force while the same particle would feel a repulsive force from a surface area of negative charge. These attractive and repulsive forces may be sufficient, at low wind speeds, to steer the particle in the plane of the surface, resulting in the oscillating travel of the snowsnake.

Including electrostatic force in the equations of motion for saltating particles could also help interpret wind-tunnel studies, where large electrostatic charge 
build-up occurs when particles are introduce, and may offer some insight into roadway and runway icing conditions in ground blizzards. Measurements of electricfield strength in the snow saltation layer are needed to assess the validity of these trajectory computations. While this analysis is directed toward saltation of snow, the approach should also be valid for sand saltation in air, where electrostatic charges also develop.

\section{CONCLUSIONS}

In blowing and drifting snow, electric fields normal to the surface exceed fair-weather potential gradients by several orders of magnitude, according to reported measurements. Other reports show saltating snow particles develop large charge-to-mass ratios. These charges develop, at least in part, through charge separation by impacts with surface particles, but the overall charging mechanisms are not clear from the literature. The effect these charges have on saltation trajectories depends on the strength of the electric field very near the surface. Estimates of the field strength in the saltation region, assuming a bed of ice spheres with charge-to-mass ratio equal to values measured on saltating snow particles, range as high as $500 \mathrm{kV} \mathrm{m}^{-1}$. In such fields, computed trajectories of charged particles vary significantly from computations without charge. These results call for experiments to determine the electric field in the saltation layer, very near the surface boundary.

\section{ACKNOWLEDGEMENT}

The authors wish to thank Dr R. A. Schmidt, U.S. Forest Service, for many helpful ideas and suggestions in the formulation of this problem, and review of the manuscript.

\section{REFERENGES}

Barré, M. 1953. Propriétés électriques du blizzard. Ann. Géophys., 9(2), 164-166.

Bagnold, R.A. 1941. The physics of blowing sand and desert dunes. London, Methuen.

British National Committee for the Polar Year. 1937. British Polar Year Expedition, Fort Rae, N.W. Canada, 1932-1933. Volume 1. Discussion of results. London, Royal Society.

Gilbert, K. G. 1914. The transportation of débris by running water. U.S. Geol. Surv. Prof. Pap. 86.

Henry, P.S. H. 1953. The role of asymmetric rubbing in the generation of static electricity. Br. J. Appl. Phys., Supplement 2, S31-S36.

Hobbs, P. V. 1974. Ice physics. Oxford, Clarendon Press.

Kikuchi, T. 1981. A wind tunnel study of the aerodynamic roughness associated with drifting snow. Cold Reg. Sci. Technol., 5(2), 107-118.

Kobayashi, D. 1972. Studies of snow transport in lowlevel drifting snow. Contrib. Inst. Low. Temp. Sci., Ser. A 24.

Latham, J. 1963 Electrification produced by the asymmetric rubbing of ice on ice. Br. F. Appl. Phys., 14(8), 488-490.

Latham, J. and B.J. Mason. 1961. Electric charge transfer associated with temperature gradients in ice. Proc. R. Soc. London, Ser. A, 260(1303), 523-536.

Latham, J. and A.H. Miller. 1965. The role of ice specimen geometry and impact velocity in the Reynolds-Brook theory of thunderstorm electrification. F. Atmos. Sci., 22(5), 505-508.

Latham, J. and J. Montagne. 1970. The possible importance of electrical forces in the development of cornices. F. Glaciol., 9(57), 375-384.

Latham, J. and C.D. Stow. 1965. Electrification produced by collision between ice crystals and soft hail pellets in a thunderstorm. Q. J. R. Meteorol. Soc., 91(387), 99-100.

Latham, J. and C.D. Stow. 1967. A laboratory investigation of the electrification of snowstorms. $Q$. 7. R. Meteorol. Soc., 93(395), 55-68.

Maeno, N., K. Araoka, K. Nishimura and Y. Kaneda. 1979. Physical aspects of the wind-snow interaction in blowing snow. 7. Fac. Sci., Hokkaido Univ., Ser. VII, 6(1), 127-141.

Maeno, N. and 8 others. 1985. Wind-tunnel experiments on blowing snow. Ann. Glaciol., 6, 63-67.

Magono, C. and K. Sakurai. 1963. On the electric charge of drifting snow pellets. F. Meteorol. Soc. Jpn, 41, 211217.

Pearce, D. C. and B. W. Currie. 1949. Some qualitative results on the electrification of snow. Can. F. Res., $27 \mathbf{A}(1), 1-8$.

Reynolds, S.E., M. Brook and M.F. Gourley. 1957. Thunderstorm charge separation. F. Meteorol., 14, 426436.

Schaefer, V.J. 1947. Properties of particles of snow and the electrical effects they produce in storms. Trans. Am. Geophys. Union, 28(4), 587-592.

Schmidt, R. A. 1980. Threshold wind-speeds and elastic impact in snow transport. F. Glaciol., 26(94), 453-467.

Schmidt, R.A. 1981. Estimates of threshold windspeed from particle sizes in blowing snow. Cold Reg. Sci. Technol., 4(3), 187-193.

Scott, W.D. and P. V. Hobbs. 1968. The spectra of charging events due to the collision of natural ice particles with an ice surface. Q. J. R. Meteorol. Soc., 94, 510-533.

Scott, W. D. and Z. Levin. 1970. The effect of potential gradient on the charge separation during interactions of snow crystals with an ice sphere. 7. Atmos. Sci., 27(3), $463-473$.

Simpson, G. C. 1919. British Antarctic Expedition, 1910-1913. Meteorology. Volume 1. Discussion. Calcutta, printed by Thacker, Spink and Co.

White, B. and J. Schultz. 1977. Magnus effects in saltation. J. Fluid Mech., 81(3), 495-512.

Wishart, E. R. 1970. Electrification of Antarctic drifting snow. International Association of Scientific Hydrology Publication 86 (Symposium at Hanover 1968 Glaciological Exploration), 316-324.

Wishart, E. R. and U. Radok. 1967. Electrostatic charging of aerial wires during Antarctic blizzards. Polar Meteorology. Technical Note 87, 492-529. (WMONo. 211.TP.111.)

The accuracy of references in the text and in this list is the responsibility of the authors, to whom queries should be addressed. 